\title{
Are Rotten Apples Edible? Challenging Commonsense Inference Ability with Exceptions
}

\author{
Nam Do \\ Brown University \\ Providence, RI, USA \\ nam_dodalumni.brown . edu
}

\author{
Ellie Pavlick \\ Brown University \\ Providence, RI, USA \\ ellie_pavlick@brown.edu
}

\begin{abstract}
Previous studies have argued that pre-trained language models encode commonsense relational knowledge (e.g. that apples are edible). However, simultaneous work has revealed that such models are often insensitive to context, even ignoring overt contextual cues such as negations. In this paper, we investigate whether masked language models (the BERT family) can move beyond naive associative biases (e.g., apple $\rightarrow$ edible) when the context warrants (e.g. ranking inedible higher when presented with the information that the apple is rotten). We introduce the WinOVENTI procedure, which adversarially exploits generic associations in masked language models to create model-specific Winograd-style entailment schemas. Using our constructed WinOVENTI challenges set of over 2,000 schemas, we show that language models in the BERT family experience a steep drop in performance on prompts that require them to pick answers which require reasoning about context (e.g., from $89.8 \%$ to $18.4 \%$ for BERT $_{\text {LARGE}}$ ). We present evidence that language models exhibit different associative biases, suggesting a need for future work in developing and analyzing frameworks similar to WINOVENTI that are tuned to model-specific weaknesses.
\end{abstract}

\section{Introduction}

Humans exhibit commonsense knowledge through their ability to identify generics (e.g., that a dog has four legs) while still recognizing that exceptions to such rules are possible (e.g., that there are cases of three-legged dogs) (Greenberg, 2007), and that the probability of such exceptions can vary based on the context (e.g. "the dog is running" vs. "the dog is hobbling"). A prerequisite to comparing a machine's performance to human intelligence is, hence, the verification that machines can exhibit a sensitivity to context that would allow them to perform as well on cases that require reasoning about exceptions as on cases that require recalling generic associations.

Recent work (Petroni et al., 2019) has shown that large pretrained language models, in particular Masked Language Models (MLMs) such as BERT (Devlin et al., 2018) are competent at associating entities with their common characteristics. For example, BERT $_{\text {LARGE }}$ readily recalls apple $\rightarrow$ edible and charcoal $\rightarrow$ hot. However, as demonstrated by Ettinger (2020) and Kassner and Schütze (2019), BERT is insensitive to various overt contextual cues, notably negation. For example, given the context "The shower is _," BERT $\mathrm{LARGE}_{\text {L }}$ predicts the words "cold", "long", and "empty", the same top 3 predictions it makes given the context "The shower is not __. Such results suggest that while language models like BERT capture many commonsense patterns, such success might be limited to inferences involving common generalizations (appearing in an affirmative context, or using common lexical associations) and not those involving exceptions (appearing in a negative context, or requiring the models to choose less frequently associated lexical items).

In this paper, we investigate whether it is indeed the case that the "commonsense reasoning" exhibited by MLMs is limited to frequent generalizations as opposed to exception cases. We make three main contributions. First, we present the WinOVENTI procedure (\$2) for identifying model-specific associative biases and adversarially building Winogradstyle challenges (Levesque et al., 2012) to test models' commonsense inference ability. Second, we apply the WINOVENTI procedure to evaluate the commonsense inference performance of a suite of pre-trained MLMs (§3). We find that all the evaluated models experience dramatic performance drops on prompts that require the models to reason about exceptions to commonsense generaliza- 
WinoVenti Procedure Summary

Step 1: Identifying Generic Associations
Sample In: $\{$ honey, tarantula $\}$
Sample Out (BERT LARGE): honey $\rightarrow$ good,
tarantula $\rightarrow$ poisonous,
tarantula $\rightarrow$ female
See Table 3

Step 2: Collecting Exceptions of Associations

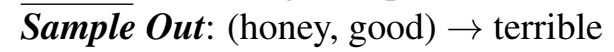

(tarantula, poisonous) $\rightarrow$ safe

(tarantula, female) $\rightarrow$ male

Step 3: Adversarial Filtering

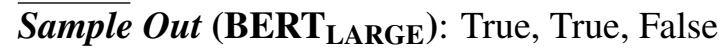

Rationale: In the last example, $\mathrm{BERT}_{\mathrm{LARGE}}$

associates the exception characteristic (male)

with the entity (tarantula) more strongly than

the generic association (female).

Step 4: Collecting Premises

Sample Prompt:

(1) The honey is [good/terrible].

(2) The tarantula is [poisonous/safe].

Sample Out: (1) After adding honey to my tea, it was (delicious/oversweet).

(2) Kim was (terrified/thrilled) when he asked her to hold the tarantula.

Step 5: Challenge Set Validation

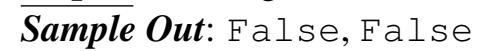

Rationale: In the first example, word association can be used to select the correct answer. In the second, the property-to-association mapping is not one-to-one, causing ambiguity.

Table 1: Summary of the WINOVENTI pipeline for constructing common sense inference challenge set.

tions. Humans, in contrast, perform consistently well $(\sim 90 \%)$ across inferences in our data. Third, we release our human-curated evaluation dataset of 2,176 sentence pairs probing inferences about commonsense generalizations and exceptions. All of our code and data are available at http://http: // commonsense-exception.github.io.

\section{WinoVenti Procedure}

\subsection{Overview}

The WinoVenti procedure aims to produce Winograd-style sentence pairs to test models' ability to reason about common sense generics and exceptions. For example, a sentence pair might look like the following:

$s_{g}$ : Zeke says that the apple is delicious. The apple is [MASK]. $\rightarrow$ edible $>$ inedible

$s_{e}$ : Zeke says that the apple is rotten. The apple is [MASK]. $\rightarrow$ inedible $>$ edible

That is, we seek to generate pairs of sentenceswhich we call $s_{g}$ and $s_{e}$, for generic and exceptionwhich differ by a single word $\left(w_{g} / w_{e}\right)$, such that that difference should lead to a change in the relative probability of other words $\left(o_{g} / o_{e}\right)$ in the context. For example, the presence of $w_{e}=$ "rotten" causes $o_{e}=$ "inedible" to be more probable given $s_{e}$ than given $s_{g}$. We seek to generate such pairs adversarially, meaning that, in the example above, we want to ensure that the model generally associates "edible" with "apple" and thus performing correctly on $s_{e}$ requires using context to override this prior association.

Our five-step procedure is summarized in Table 1. First (\$2.2), given a model and a target noun, we identify the model's generic associations (or just generics), i.e., the characteristics that the model tends to associate with the noun in a generic context. For example, given a target noun "apple", we might identify "edible" as one such association. Second ( $\$ 2.3)$, crowd workers are asked to provide contrasting characteristics (or exceptions) that could plausibly describe the noun. For example, workers might provide "inedible" or "plastic" as characteristics that contrast with "edible" in the context of "apple". Third (\$2.4), we perform an adversarial post-processing step in which we filter out worker-provided exceptions that the model associates with the target more strongly than the original generic. That is, if a worker provides the characteristic "poison" and it turns out that the model associates "poison" with "apple" more strongly than it associates "edible" with "apple", we would filter "poison" out of our list of exceptions. Fourth (\$2.5), we crowdsource premise pairs that would ideally effect the relative probability of the generic characteristic vs. the exception characteristic, such as those shown in $s_{g}$ and $s_{e}$ above. Finally (\$2.6), the schemas are validated by human annotators, after which we filter out annotations that are trivial or ambiguous. Using this five-step procedure, we construct the WINOVENTI BERT LARGE challenge set of 2,176 sentence pairs. ${ }^{1}$

\footnotetext{
${ }^{1}$ Available at: http://commonsense-exception.github.io.
} 


\begin{tabular}{|ll|}
\hline Prompt pairs & Generic/Exception Outcomes \\
\hline$s_{g}=$ Regina screamed when she picked up the pan. The pan is _. & hot / cold \\
$s_{e}=$ Regina shivered when she picked up the pan. The pan is _. & hot / cold \\
\hline$s_{g}=$ Tonight Mike's pets would be happy. The pet food is __. & available / unavailable \\
$s_{e}=$ Tonight Mike's pets would be hungry. The pet food is _. & available / unavailable \\
\hline
\end{tabular}

Table 2: Examples of our schemas. Each prompt contains a premise with an underlined special word (first sentence) and an outcome sentence. $s_{g}=$ generic prompt. $s_{e}=$ exception prompt. All prompts and exception outcomes are crowdsourced, whereas generic outcomes are generic associations identified in some MLM $\left(\mathrm{BERT}_{\mathrm{LARGE}}\right.$, in our paper). Correct answers are in bold.

We draw our target nouns from the THINGS dataset (Hebart et al., 2019) which consists of 1,854 concepts from 27 semantic categories. All of our crowdsourcing tasks are run on Surge (surgehq. ai), a high-quality crowdsourcing platform similar to Amazon Mechanical Turk. For the exception and premise generation stages ( $\$ 2.3$ and $\S 2.5$ ), we recruited 100 workers through a qualification task that required the workers to walk through the entire WINOVENTI pipeline. The validation annotation (§2.6) could be performed by anyone on the Surge platform. The pay per response for the crowdsourcing stages is as follows, chosen based on difficulty and time taken to complete the task: (1) exception generation (\$2.3): $\$ 0.1$, (2) premise generation (\$2.5): $\$ 0.3$, (3) challenge set validation (\$2.6): $\$ 0.2$, and (4) human performance: $\$ 0.03$.

\subsection{Step 1: Identifying Generic Associations}

Given a set of target nouns, our first step is to find a set of generic associations which models associate with the noun regardless of context. To do this, we focus on the widely-used BERT LARGE $_{\text {model }}$ (Devlin et al., 2018), specifically the HuggingFace cased, whole word masking implementation (24layer, 1024 hidden dimension, 16 attention heads, $336 \mathrm{M}$ parameters). We base our procedure off of the insight from Ettinger (2020), which demonstrated BERT's insensitivity to negation. For example, given the contexts "A robin is (not) a [MASK]," BERT's top two predictions would share $\{$ bird, robin $\}$ in common, or given the contexts "A daisy is (not) a [MASK]," BERT's top three predictions would share $\{$ daisy, rose, flower $\}$ in common. Our experiments show that this behavior holds consistent for other MLMs such as RoBERTa (Liu et al., 2019), ALBERT (Lan et al., 2019), Longformer (Beltagy et al., 2020), SqueezeBERT (Iandola et al., 2020), and MobileBERT (Sun et al., 2020). Thus, to identify context-invariant associations, we feed in both affirmative and negated templates ${ }^{2}$ of the form "The [ENTITY] is (not) [MASK]". We then define the models generic associations to be the set of words that are in common between the top- $k$ predictions in the affirmative context and the top- $k$ predictions in the negative context. We experiment with $k \in\{1,3,5,8\}$. Table 3 shows generic associations generated for BERT $\mathrm{LARGE}_{\text {in }}$ this way for different values of $k$.

\begin{tabular}{|cl|}
\hline$k$ & Generic Associations for BERT LARGE $_{\text {A }}$ \\
\hline 1 & $\begin{array}{l}\text { desk } \rightarrow \text { empty, tarantula } \rightarrow \text { poisonous, } \\
\text { couch } \rightarrow \text { comfortable, syrup } \rightarrow \text { sweet, } \\
\text { honey } \rightarrow \text { sweet, compass } \rightarrow \text { accurate }\end{array}$ \\
\hline $3 \quad \begin{array}{l}\text { desk } \rightarrow \text { there, tarantula } \rightarrow \text { edible, } \\
\text { couch } \rightarrow \text { empty, syrup } \rightarrow \text { bitter, } \\
\text { honey } \rightarrow \text { good, compass } \rightarrow \text { true }\end{array}$ \\
\hline $5 \quad \begin{array}{l}\text { desk } \rightarrow \text { full, tarantula } \rightarrow\{\text { female, male }\}, \\
\text { couch } \rightarrow \text { warm, syrup } \rightarrow \text { edible } \\
\text { honey } \rightarrow \text { edible, compass } \rightarrow \text { right }\end{array}$ \\
\hline $8 \begin{array}{l}\text { desk } \rightarrow\{\text { mine, clean }\}, \text { tarantula } \rightarrow \text { small } \\
\text { couch } \rightarrow\{\text { clean, big }\}, \text { syrup } \rightarrow \text { orange, } \\
\text { honey } \rightarrow\{\text { delicious, there, honey }\} \\
\text { compass } \rightarrow \text { wrong }\end{array}$ \\
\hline
\end{tabular}

Table 3: Examples of BERT LARGE $_{\text {generic associations }}$ for different values of $k$. Generic associations are cumulative across different $k$ values (e.g., in the end, we have compass $\rightarrow$ accurate, true, right, wrong $\}$ ).

How similar are associations across models? Since our dataset is constructed in particular to be adversarial against BERT LARGE$_{\text {, it is relevant to }}$ ask whether such a dataset will be equally adversarial against all models in the BERT family. To quantify whether models in the BERT family (described in §3.2) differ in the generic associations

\footnotetext{
${ }^{2}$ We use a simple set $S$ of two templates that differ in the ending punctuation $(S=\{$ "The [ENTITY] is [MASK].' ', ' The [ENTITY] is $[M A S K], '$,$\} ).$
} 


\begin{tabular}{|l|lll|}
\hline Model name & $\mathbf{k = 1}$ & $\mathbf{k}=\mathbf{3}$ & $\mathbf{k}=\mathbf{5}$ \\
\hline BERT $_{\text {BASE }}$ & 0.59 & 0.26 & 0.17 \\
BERT $_{\text {LARGE }}$ & 1 & 1 & 1 \\
RoBERTa $_{\text {SMALL }}$ & 0.61 & 0.2 & 0.11 \\
RoBERTa $_{\text {LARGE }}$ & 0.57 & 0.19 & 0.12 \\
DistilRoBERTa $_{\text {DistilBERT }}$ & 0.53 & 0.15 & 0.08 \\
Distilie & 0.57 & 0.18 & 0.12 \\
SqueezeBERT & 0.6 & 0.23 & 0.13 \\
MobileBERT & 0.65 & 0.28 & 0.16 \\
ALBERT $_{\text {BASE }}$ & 0.42 & 0.07 & 0.04 \\
ALBERT $_{\text {LARGE }}$ & 0.51 & 0.1 & 0.07 \\
ALBERT $_{\text {XLARGE }}$ & 0.59 & 0.18 & 0.08 \\
ALBERT $_{\text {XXLARGE }}$ & 0.48 & 0.13 & 0.07 \\
\hline
\end{tabular}

Table 4: Jaccard similarity of Generic Associations made by models with respect to BERT $\mathrm{LARGE}_{\text {. The }}$ closer to 1 , the more similar.

identified using this procedure, we compute the Jaccard similarity-i.e., $\frac{|A \cap B|}{|A \cup B|}$, with A and B being the set of associations made by two models. Table 4 shows the results for $k=1,3$, and 5 . BERT BASE $_{\text {B }}$

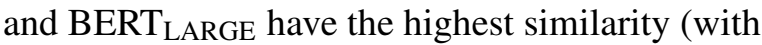
a mean Jaccard score of 0.72). Most other models only share roughly half of the generic associations with another model. As $k$ increases, generic associations start to differ across models (signified by a decrease in the Jaccard similarity score). This signifies that models differ significantly in the generic associations that they make.

Qualitatively, associations made by BERTfamily, RoBERTa-family and other models differ from one another. While the evaluated RoBERTafamily models display a slight tendency to associate the words "empty", "broken", "dead" with many nouns, the BERT-family models tend to make other associations such as "used", and "edible". ALBERT models, on the other hand, make quite different associations from all other models, such as "adjustable", "gone", "a", and "covered" (with a full $41 \%$ of the associations made by ALBERT XXLARGE being “adjustable”).

As we base this study on the associations made by BERT LARGE$_{\text {, future work is to be done to extract }}$ the different associations made by other MLMs to pose a harder and richer set of challenges for those models.

\subsection{Step 2: Crowdsourcing Exceptions to Generic Associations}

Given the identified associative biases, we then use crowdsourcing to find alternative characteristics that can be true of the noun, but are perhaps less stereotypically associated (e.g., "apples" can be "inedible" if, for example, they are made of plastic, or are poisoned). Workers are given statements of the form "The [NOUN] is [PROPERTY]" where [PROPERTY] is one of the generic associations collected as described above (e.g., "The apple is edible"), and then is asked to provide up to three adjectives that would describe [NOUN] in the case where it is not [PROPERTY] (e.g., in the case that the "apple" is not "edible"). To increase the quality of tasks presented to workers in later stages, we also ask workers in this stage whether or not a presented statement makes sense, and filter out any sentences which workers flag as nonsensical. Of the noun-property associations generated in our first step, $10.45 \%$ are filtered out in this step.

The model-generated associative pairs can be noisy. Particularly, we note that as we increase $k$, the model becomes more likely to generate nonsense pairs (e.g., glass $\rightarrow$ glass, dart $\rightarrow$ legal, triangle $\rightarrow$ circular), and that the stereotypical strength of association decreases (as shown in Table 3). Thus, to increase the quality of the final challenge set and minimize workers' confusion, our pipeline uses two main criteria to select which association pairs to present to workers. First, associations are selected in an increasing order of $k$, meaning we would include all associations at $k=1$ first, and then those at $k=3$, and so on. Second, the templated statements presented to workers are ranked according to perplexity scores assigned to them by a unidirectional language model. For each $k$ of interest, we identify the inflection point in the perplexity score, and only retain samples for which the perplexity is below this point. At the end when we have gathered more samples than we plan to post, we perform a final round of inflection point identification and retention. This leaves us with 1990 entity-association pairs.

To construct WINOVENTIBERT LARGE, we selected 1990 entity-bias pairs to present to workers (331 pairs were of 1 -associative biases, 780 of 3 -associative biases, 825 of 5 -associative biases, and 54 of 8 -associative biases). 28 workers participated in this stage, and for each task workers were paid $\$ 0.1$. At the end, we received 2,994 valid 
exception annotations from crowd workers.

\subsection{Step 3: Adversarial Filtering}

The goal of adversarial filtering is to make sure that a model clearly favors the generic association with a target noun (identified by an MLM) over an exception association (crowdsourced) without any additionally introduced context. A triplet of (Target Noun, Generic Association, Exception Association) passes our adversarial filtering stage if the probability that the model associates the Generic Association with the Target Noun (through statements of template "The [NOUN] is _") is higher than that which the model associates with the Exception Association.

This filtering is adversarial in that, by making sure that a model innately favors one association (e.g., edible) with a target noun (e.g., apple) over another (e.g., inedible), the model has to demonstrate a strong enough sensitivity to context to select the other association over the one that it innately prefers (e.g., when presented with the information that the apple is rotten).

In the construction of WINOVENTIBERT LARGE, after adversarially filtering using BERT $_{\text {LARGE, we }}$ retained 2, 745 (Target Noun, Generic Association, Exception Association) triplets. Some examples of triplets that were filtered out (in the same format) are: (stair, long, short), or (mug, full, empty).

\subsection{Step 4: Crowdsourcing Premises}

As a result of Step 3, we have a set of (noun, generic associations, exception associations) triplets that have met our adversarial filtering criteria. We then ask workers to create a minimal pair of premise sentences $\left(s_{g} / s_{e}\right)$ differing by exactly one word $\left(w_{g} / w_{e}\right)$ which differ in whether the generic outcome $\left(o_{g}\right)$ or the exception outcome $\left(o_{e}\right)$ is the most probable continuation. For example, given the triplet ("apple”, "edible”, “inedible”), our sentence pair might be $s_{g}=$ "the apple is sweet and $s_{e}=$ "the apple is rotten. To minimize the cognitive load on crowdworkers while still communicating these requirements, we provided examples of two good and two bad premise pairs with a brief explanation each of why each was good/bad, covering all the requirements above. ${ }^{3}$ We also encouraged workers to be creative and provide premises that have diverse sentence structures. In total, 2, 745

\footnotetext{
${ }^{3}$ Our exact instructions and examples given to workers are provided in Appendix A.2
}

premise pairs were collected. Table 5 shows several examples of contexts generated in this way.

\section{Crowdsourced Premises}

Given: The mail is [anonymous/identifiable].

Annotation: I received a letter in the mail from a (stranger/friend).

Given: The pill is [safe/unsafe].

Annotation: You will feel (better/worse) if you take the pill.

Given: The paper bag is [empty/full].

Annotation: I took a (folded/heavy) bag to the store.

Given: The timer is [accurate/inaccurate].

Annotation: Jim was (early/late) to work of his timer.

Given: The bed is [comfortable/uncomfortable]. Annotation: Lola slept [soundly/miserably].

Table 5: Premise annotations collected given a target noun and the identified generic/exception associations, combined into a natural language sentence of format "The __ is __ " presented to workers.

\subsection{Step 5: Challenge Set Validation}

We evaluate each sentence pair using two criteria. First, is it the case that the contexts differentiate the probabilities of $o_{g}$ and $o_{e}$ such that $o_{g}$ only makes sense given $s_{g}$ and $o_{e}$ only makes sense given $s_{e}$. E.g., given "Matthew says that the apple is rotten. The apple is [MASK]", can edible be a sensible answer)? Second, we ensure that the special words are not synonymous with the outcomes, i.e., that $w_{g}$ and $o_{g}$ are not synonyms, nor are $w_{e}$ and $o_{e}$. If the majority of workers (out of three) judge the sentence pairs to pass the above criteria, the pair is deemed valid. Criterion 1 is to ensure that the outcomes are unambiguous to humans, while criterion 2 is to ensure that synonymy can not be used to trivially find the correct answer. For example, criterion 1 filtered out prompts such as "Mary made popsicles out of vitamins. The popsicle is [MASK]." where both the choices (edible / nutritious) could apply. Criterion 2 filtered out prompts like "The doctor used a loaded test tube. The test tube is [MASK]." where the correct answer (filled/hollow) could easily be selected using word association. Of the 2,678 prompt pairs posted for evaluation, 502 were filtered out for failing at least one of the two aforementioned criteria, leaving us with the 
final challenge set of 2,176 prompt pairs $(4,352$ prompts in total).

\subsection{WinoVenti Challenge Set}

To summarize, the final WINOVENTIBERT LARGE challenge set consists of 2,176 prompt pairs $(4,352$ challenge prompts in total) about 978 distinct entities. Each entity has at most 5 generic associations, for a total of 186 distinct generic associations identified by BERT LARGE $_{\text {across all }}$ entities. The length of premises are on average 8 words. Using SpaCy's Part of Speech tagger, we identified that the special words $\left(w_{g}\right.$ and $w_{e}$ ) across different premises are predominantly adjectives (apprx. 34\%), nouns (apprx. 30.3\%), and verbs (apprx. 22.4\%), with the presence of other parts of speech such as adverbs, adpositions, numbers, determiners, proper nouns, or pronouns. Approximately $54.5 \%$ of the premises have the special words in the last third, $28.4 \%$ in the middle third, and the rest $16.9 \%$ in the first third of the premise. The challenge set is available at http://commonsense-exception.github.io/.

\section{Experiments}

\subsection{Task Definition}

The task is defined as: given a pair of sentences $\left(s_{g}, s_{e}\right)$ with the format in Table 2, and the pair of single-word candidate outcomes $\left(o_{g}, o_{e}\right)$, does the model correctly rank $o_{g}$ as more probable than $o_{e}$ given $s_{g}$ (general test), and, symmetrically, rank $o_{e}$ as more probable than $o_{g}$ given $s_{e}$ (exception test). An MLM's performance is reported as the percentage of sentences for which the language model successfully gives the correct answer a higher probability of filling in the blank than the incorrect answer. Each model is evaluated on three different subsets of the full WINOVENTIBERT LARGE dataset, as follows: All refers simply to the entire WINOVENTIBERT LARGE challenge set (which is only adversarially filtered using BERT LARGE$_{\text {); }}$ Individual refers to a model-specific subset of WINOVENTI BERT LARGE, specifically those pairs which result after additionally model-specific adversarial filtering; Intersection refers to the set of 188 prompts (94 each for the general test and the exception test) that result when we take the intersection of each of the model-specific subsets generated described in Individual. Note that both Individual and Intersection reflect the performance of models in an adversarial setting, and we do not expect these subsets to show meaningfully different results. We include Intersection simply so that we can compare all models on a fixed test set in an apples-to-apples setting, since the Individual subsets will vary from one model to the next.

\subsection{Models}

We study the performance of the following pretrained models (HuggingFace implementation) on our WINOVENTIBERT LARGE challenge set:

BERT-family We used BERT BASE $_{\text {(cased) and }}$ BERT $_{\text {LARGE (cased, trained with a whole word }}$ masked language modeling objective) models (Devlin et al., 2018). We also evaluated DistilBERT (Sanh et al., 2019) (cased), which has $40 \%$ less parameters than BERT while still performing almost as good as the original model. MobileBERT (Sun et al., 2020), similarly seeking to compress and accelerate the BERT model, is also included in our experiments (uncased). Additionally, our experiments also evaluated SqueezeBERT (Iandola et al., 2020) (uncased), which has a similar bidirectional transformer architecture like BERT, but uses grouped convolutions instead of certain fullyconnected layers.

RoBERTa-family Our experiments also evaluate RoBERTa BASE $_{\text {and RoBERTa }}$ LARGE (Liu et al., 2019) versions that were trained on a masked modeling objective. RoBERTa build on BERT, differing in hyperparameter choices and pre-training objective. We also used DistilRoBERTa (Sanh et al., 2019), which follows the same training and distillation process as DistilBERT, but based on RoBERTa instead.

ALBERT-family We additionally evaluated ALBERT $_{\{\text {BASE, LARGE, XLARGE, XXLARGE\} }}$ models (Lan et al., 2019). Built off of BERT, ALBERT is designed to reduce the number of parameters and perform better on downstream tasks with multi-sentence inputs.

\subsection{Finetuning}

To evaluate how the models perform after being fine-tuned on datasets that contain exceptions in similar sentence structures to our challenge set, we fine-tuned a subset of the models in question (BERT BASE, RoBERTa $_{\mathrm{BASE}}$, DistilRoBERTa, SqueezeBERT, MobileBERT, and

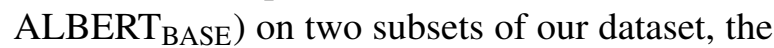
half and the full exception train sets. The half 


\begin{tabular}{|c|c|c|c|c|c|c|c|}
\hline \multirow[b]{2}{*}{ Model name } & \multicolumn{2}{|c|}{ All } & \multicolumn{3}{|c|}{ Individual } & \multicolumn{2}{|c|}{ Intersection } \\
\hline & Generic & Exceptn & Generic & Exceptn & $\mathbf{N}$ & Generic & Exceptn \\
\hline BERT $_{\text {BASE }}$ & 83.0 & 23.1 & 89.3 & 16.7 & 1871 & 96.0 & 8.3 \\
\hline BERT $_{\text {LARGE }}$ & 89.8 & 18.4 & 89.8 & 18.4 & 2176 & 97.4 & 11.4 \\
\hline RoBERTa & 79.5 & 27.2 & 87.3 & 18.8 & 1801 & 95.6 & 12.7 \\
\hline RoBERTa $_{\text {LARGE }}$ & 81.3 & 29.2 & 91.1 & 18.9 & 994 & 95.6 & 17.5 \\
\hline DistilRoBERTa & 78.3 & 26.5 & 91.1 & 13.1 & 948 & 93.9 & 14.0 \\
\hline DistilBERT & 85.8 & 19.4 & 89.8 & 15.2 & 1628 & 94.8 & 12.7 \\
\hline SqueezeBERT & 85.5 & 21.1 & 92.1 & 13.6 & 1823 & 97.4 & 7.9 \\
\hline MobileBERT & 82.7 & 23.0 & 89.8 & 15.6 & 1856 & 96.9 & 7.0 \\
\hline ALBERT $_{\text {BASE }}$ & 77.4 & 28.0 & 87.2 & 18.4 & 1525 & 94.3 & 11.0 \\
\hline ALBERT $_{\text {LARGE }}$ & 79.6 & 26.8 & 87.6 & 18.9 & 1324 & 93.9 & 12.2 \\
\hline ALBERT $_{\text {XLARGE }}$ & 80.7 & 31.9 & 89.0 & 23.5 & 777 & 96.1 & 13.1 \\
\hline ALBERT $_{\text {XXLARGE }}$ & 77.7 & 32.4 & 80.0 & 32.4 & 959 & 83.8 & 31.9 \\
\hline "Human performance & $\overline{91.1}$ & $\overline{90.2}$ & - & - & - & - & - \\
\hline
\end{tabular}

Table 6: Models' performance on our generic and exception prompts. Generic tests evaluate whether, given a generic prompt, the model would rank the generic outcome $o_{g}$ higher than the exception $o_{e}$ ). Exceptn tests similarly check if the exception outcome $o_{e}$ is ranked higher than the generic $o_{g}$ given an exception context.

exception set is created by selecting half of the WINOVENTI BERT LARGE challenge set (2176 out of 4352 schemas). $50 \%$ of the training schemas (1088 out of 2176) are selected to be generic schemas, and the rest are exception schemas. With this configuration, models are fine-tuned on both generic and exception schemas, and are sequentially evaluated on unseen challenges (with a similar distribution of $50 \%$ generic schemas and $50 \%$ exception).

With the full exception train set, models are finetuned exclusively on exception schemas. From the set of exception challenges (2176 schemas), we performed a 80-20 train-test split to select the full exception train set. To evaluate models trained on the full exception train set, in addition to evaluating the model accuracy on the held out exceptions (20\% 435 of the 2176 exception schemas), we also evaluate the fine-tuned models' on (1) all the generic challenges (2176 schemas), and (2) on a test set similar to the above where half of the schemas are generic challenges and another half are unseen exception challenges ( 870 schemas). The different test scenarios help us understand how finetuning on exception challenges influences models' performance on not only unseen exception challenges, but also on generic challenges.

\subsection{Results and Analysis}

Table 6 shows each model's performance across each data subset (All, Individual, and Intersection) broken down by task (i.e., generic vs. exception test). Across the board, models perform significantly better on the generic tests than on the exception tests (where accuracies are well below the random baseline of 50\%). This provides strong evidence that models do not truly encode "common sense", but rather simply recall generic associations in a context-agnostic manner.

Looking closely at the results on the All subset, we see that models' performance on the generic test is overall lower and the performance drop on the exception test is less dramatic, compared to the results on the Individual and Intersection subsets. This trend is expected, since, after adversarial filtering, each model is only evaluated on prompts where the model is inherently (before being introduced to any additional context) skewed towards choosing the generic association as a description of the target noun. Even so, the numbers on this All set are informative: they emphasize that models' apparent success at recalling "commonsense" associations is likely largely driven by artifact. That is, when assessed on a set of common sense inferences that don't necessarily involve words from the top of a model's distribution in a given context, performance is quite poor. On inspection, we see that, for models outside the BERT family, the poor performance is often attributable to low probabilities for both the generic and exception outcomes $\left(o_{g}\right.$ and $o_{e}$ ) in both contexts, meaning the difference between the probabilities is often small. In other words, these models don't encode the same generic 
associations that BERT $\mathrm{LARGE}_{\mathrm{L}}$ encodes and, moreover, don't encode much difference at all between $o_{g}$ and $o_{e}$.

Error Analysis Looking closely at specific prompts on which models succeed and fail reveals some interesting trends. For each model, we look at the top 20 sentences from the exception test on which the model exhibited the largest errors, where the size of the error is measured by the difference in the probability that the model assigns to the incorrect outcome (in this case $o_{g}$ ) compared to the correct outcome $\left(o_{e}\right)$. For BERT LARGE $_{\text {, we find that }}$ $55 \%$ of these top 20 failures involve strong generic associations (i.e., those generated with $k=1$ ). A full $65 \%$ are cases when $o_{g}$ is "empty", suggesting that $\mathrm{BERT}_{\text {LARGE }}$ prefers to assign high probability to this word across all contexts (see §2.2). In contrast, looking at the top 20 sentences on which BERT $_{\text {LARGE }}$ performed best, we learn that only one involves $o_{g}=$ "empty", and only four involve strong generic associations $(k=1)$.

Performing similar analysis for the other BERTfamily models (BERT SMALL $_{\text {, DistilBERT, Mobile- }}$ BERT, SqueezeBERT), we see that the majority of the successes involve non-associative nouncharacteristic pairs (i.e., pairs where the characteristic is not identified as a generic association with the noun by our procedure described in Section 2.2). For BERT SMALL $_{\text {and DistilBERT, } 40 \% \text { of their } 20}$ most successful cases involved nouns for which the models did not encode any generic associations. For SqueezeBERT and MobileBERT, it is $55 \%$ and $60 \%$, respectively. This may signify stronger a relationship between a model not identifying a generic association with a target noun and that model being sensitive to a change in context about that target noun.

Fine-tuning Analysis The results of our finetuning experiments are shown in Figure 1 (with additional results in §A.1). We see that, in general, fine-tuning models on a dataset that contains exceptions (where the challenge format remains the same between the train and test sets) can increase the performance on unseen exceptions, but does so at the expense of performance on generic prompts. Specifically, when we train on a mix of generic and exception schemas (our half exception set), the model improves only slightly in performance on exceptions, and converges to the same trend as the un-finetuned model: i.e., performance on generics far exceeds that on exceptions. In contrast, when we train on only exception schemas (our full exception set), the performance on unseen exception challenges increases faster and more significantly, but this increase is at the expense of the rapid decrease of performance on generic challenges.

This poor performance on exceptions (at the expense of their performance on generics), suggests that the conceptual associations encoded by MLM models is fairly shallow: even with finetuning, the models are not able to differentiate these types of context-specific associations in a way that allows them to perform well on both types of inferences simultaneously. Future work is needed to develop models with different architectures or loss functions that might be capable of encoding more nuanced conditional associations.

\section{Related Work}

Ettinger et al. (2017) bring up the fundamental problem of NLP models ignoring rare language phenomena, as they typically rely on independently and identically distributed probably-approximatelycorrect model of learning, and as they often use overly simplistic loss functions. Complementary to our project, Ettinger et al. (2017) encourage robust error analysis of NLP systems through developing challenges that are based on linguistic phenomena, and that have a low barrier to entry.

Common sense and probing. NLP has been interested in encoding commonsense relations for a long time (Liu and Singh, 2004). Recent work has shown how pre-trained LMs exhibit common sense knowledge even before fine-tuning (Petroni et al., 2019), and that they can be built and used to mine more commonsense information (Bosselut et al., 2016; Davison et al., 2019). While this signifies how LMs encode some common sense and prototypical properties of nouns (Weir et al., 2020), many researchers are pointing out these models' insensitivity to context (Ettinger, 2020; Ravichander et al., 2020), which is antithetical to common sense.

Challenge Sets Many existing challenge sets have provided concrete frameworks to evaluate models inference ability, through coreference resolution (notably Winograd Schema Challenge WSC (Levesque et al., 2012)) or pronoun resolution (notably in PDP (Morgenstern et al., 2016)). In this work, similar to the Winograd Schemas (Levesque 

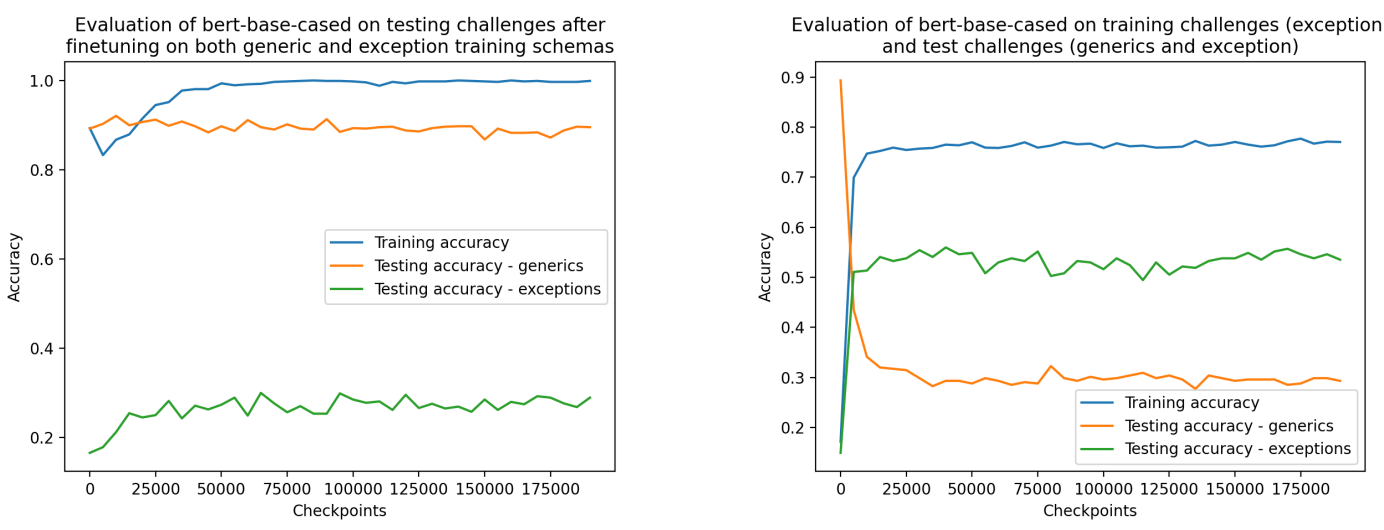

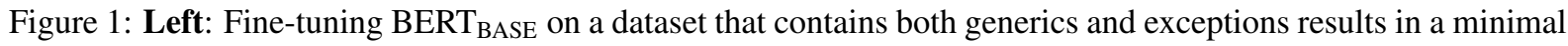
increase in performance on exceptions. Right: Fine-tuning BERT $\mathrm{BASE}_{\mathrm{B}}$ on a dataset containing only exceptions results in an increase in performance on exceptions, at the expense of the accuracy on generics.

et al., 2012), we also generate pairs with a similalr structure (pairs of premises that differ in one word that would determine the answer). However, while Winograd schemas are model-agnostic, our approach factors in models' behavior in the design of schemas in order to guarantee models' bias towards one specific answer (for each prompt).

Sakaguchi et al. (2020) build the WINOGRANDE adversarial challenge set through using language models to detect and filter out schemas with language-based biases that would trivialize the task of picking the correct answer. WINOGRANDE aims to minimize the chance of models getting the right answers for the wrong reasons (through leveraging simple lexical associations that are annotation artifacts by human annotators). Our key motivation, meanwhile, is to adversarially leverage the biases that models associate with entities to "trick" them into choosing incorrect answers. Our work uses adversarially constructed test sets to expose heuristics that models use. This technique has been used widely in probing/analysis work, e.g., (Glockner et al., 2018; Naik et al., 2018; Jia and Liang, 2017; Nie et al., 2019; McCoy et al., 2019). The idea of improving models performance on "exceptions" to "generalizations" also shares much in common with work on bias and fairness in NLP (Rudinger et al., 2018; Zhao et al., 2018, 2019).

Gardner et al. (2020) propose the development of contrast sets, which can be developed by manually perturbing existing datasets in small but meaningful ways that would change the gold label. Our work, in contrast, factor in models' insensitive associations into the construction of challenges in addition to a slight change in context that is lever- aged by contrast sets. Kaushik et al. (2019) similarly propose using counterfactually-augmented data to make models more robust against spurious associations. Our work adds to this work by demonstrating that fine-tuning on exception challenges can increase the performance of models on tail cases at the expense of the performance on generic prompts.

\section{Conclusion}

We present the WinOVENTI procedure, which adversarially exploits generic associations in masked language models to create model-specific Winograd-style schemas. Using our constructed WINOVENTI BERT LARGE challenge set, we test whether MLMs can move beyond their naive associations to select the more likely outcomes depending on the input context. We find a steep drop in models' performance on our challenges that require a sensitivity to context. We present evidence that generic associations differ from one model to another, highlighting the need for other modelspecific challenge sets that are tuned to associative biases of models other than BERT LARGE $_{\text {, and to (2) }}$ develop and analyze frameworks like WiNOVENTI.

\section{Acknowledgments}

We thank Charlie Lovering, and the members and friends of the Brown University LUNAR Lab for all the invaluable conversations, proofreading, and feedback. We thank Edwin Chen (Surge) for their support with our crowdsourcing tasks. 


\section{References}

Iz Beltagy, Matthew E Peters, and Arman Cohan. 2020. Longformer: The long-document transformer. arXiv preprint arXiv:2004.05150.

Antoine Bosselut, Jianfu Chen, David Warren, Hannaneh Hajishirzi, and Yejin Choi. 2016. Learning prototypical event structure from photo albums. In Proceedings of the 54th Annual Meeting of the Association for Computational Linguistics (Volume 1: Long Papers), pages 1769-1779, Berlin, Germany. Association for Computational Linguistics.

Joe Davison, Joshua Feldman, and Alexander Rush. 2019. Commonsense knowledge mining from pretrained models. In Proceedings of the 2019 Conference on Empirical Methods in Natural Language Processing and the 9th International Joint Conference on Natural Language Processing (EMNLPIJCNLP), pages 1173-1178, Hong Kong, China. Association for Computational Linguistics.

Jacob Devlin, Ming-Wei Chang, Kenton Lee, and Kristina Toutanova. 2018. Bert: Pre-training of deep bidirectional transformers for language understanding. arXiv preprint arXiv:1810.04805.

Allyson Ettinger. 2020. What bert is not: Lessons from a new suite of psycholinguistic diagnostics for language models. Transactions of the Association for Computational Linguistics, 8:34-48.

Allyson Ettinger, Sudha Rao, Hal Daumé III, and Emily M Bender. 2017. Towards linguistically generalizable nlp systems: A workshop and shared task. arXiv preprint arXiv:1711.01505.

Matt Gardner, Yoav Artzi, Victoria Basmov, Jonathan Berant, Ben Bogin, Sihao Chen, Pradeep Dasigi, Dheeru Dua, Yanai Elazar, Ananth Gottumukkala, et al. 2020. Evaluating models' local decision boundaries via contrast sets. In Proceedings of the 2020 Conference on Empirical Methods in Natural Language Processing: Findings, pages 1307-1323.

Max Glockner, Vered Shwartz, and Yoav Goldberg. 2018. Breaking NLI Systems with Sentences that Require Simple Lexical Inferences. In Proceedings of the 56th Annual Meeting of the Association for Computational Linguistics (Volume 2: Short Papers), pages 650-655. Association for Computational Linguistics.

Yael Greenberg. 2007. Exceptions to generics: Where vagueness, context dependence and modality interact. Journal of Semantics, 24(2):131-167.

Martin N Hebart, Adam H Dickter, Alexis Kidder, Wan Y Kwok, Anna Corriveau, Caitlin Van Wicklin, and Chris I Baker. 2019. Things: A database of 1,854 object concepts and more than 26,000 naturalistic object images. PloS one, 14(10):e0223792.

Forrest N Iandola, Albert E Shaw, Ravi Krishna, and Kurt W Keutzer. 2020. Squeezebert: What can computer vision teach nlp about efficient neural networks? arXiv preprint arXiv:2006.11316.

Robin Jia and Percy Liang. 2017. Adversarial examples for evaluating reading comprehension systems. In Proceedings of the 2017 Conference on Empirical Methods in Natural Language Processing, pages 2021-2031. Association for Computational Linguistics.

Nora Kassner and Hinrich Schütze. 2019. Negated and misprimed probes for pretrained language models: Birds can talk, but cannot fly. arXiv preprint arXiv:1911.03343.

Divyansh Kaushik, Eduard Hovy, and Zachary C Lipton. 2019. Learning the difference that makes a difference with counterfactually-augmented data. arXiv preprint arXiv:1909.12434.

Zhenzhong Lan, Mingda Chen, Sebastian Goodman, Kevin Gimpel, Piyush Sharma, and Radu Soricut. 2019. Albert: A lite bert for self-supervised learning of language representations. arXiv preprint arXiv:1909.11942.

Hector Levesque, Ernest Davis, and Leora Morgenstern. 2012. The winograd schema challenge. In Thirteenth International Conference on the Principles of Knowledge Representation and Reasoning. Citeseer.

Hugo Liu and Push Singh. 2004. Conceptnet-a practical commonsense reasoning tool-kit. BT technology journal, 22(4):211-226.

Yinhan Liu, Myle Ott, Naman Goyal, Jingfei Du, Mandar Joshi, Danqi Chen, Omer Levy, Mike Lewis, Luke Zettlemoyer, and Veselin Stoyanov. 2019. Roberta: A robustly optimized bert pretraining approach. arXiv preprint arXiv:1907.11692.

R Thomas McCoy, Ellie Pavlick, and Tal Linzen. 2019. Right for the wrong reasons: Diagnosing syntactic heuristics in natural language inference. arXiv preprint arXiv:1902.01007.

Leora Morgenstern, Ernest Davis, and Charles L Ortiz. 2016. Planning, executing, and evaluating the winograd schema challenge. AI Magazine, 37(1):50-54.

Aakanksha Naik, Abhilasha Ravichander, Norman Sadeh, Carolyn Rose, and Graham Neubig. 2018. Stress Test Evaluation for Natural Language Inference. In Proceedings of the 27th International Conference on Computational Linguistics, pages 2340 2353. Association for Computational Linguistics.

Yixin Nie, Adina Williams, Emily Dinan, Mohit Bansal, Jason Weston, and Douwe Kiela. 2019. Adversarial nli: A new benchmark for natural language understanding. arXiv preprint arXiv: 1910.14599.

Fabio Petroni, Tim Rocktäschel, Patrick Lewis, Anton Bakhtin, Yuxiang Wu, Alexander H Miller, and Sebastian Riedel. 2019. Language models as knowledge bases? arXiv preprint arXiv:1909.01066. 
Abhilasha Ravichander, Eduard Hovy, Kaheer Suleman, Adam Trischler, and Jackie Chi Kit Cheung. 2020. On the systematicity of probing contextualized word representations: The case of hypernymy in BERT. In Proceedings of the Ninth Joint Conference on Lexical and Computational Semantics, pages 88-102, Barcelona, Spain (Online). Association for Computational Linguistics.

Rachel Rudinger, Jason Naradowsky, Brian Leonard, and Benjamin Van Durme. 2018. Gender bias in coreference resolution. In Proceedings of the 2018 Conference of the North American Chapter of the Association for Computational Linguistics: Human Language Technologies, Volume 2 (Short Papers), pages 8-14, New Orleans, Louisiana. Association for Computational Linguistics.

Keisuke Sakaguchi, Ronan Le Bras, Chandra Bhagavatula, and Yejin Choi. 2020. Winogrande: An adversarial winograd schema challenge at scale. In Proceedings of the AAAI Conference on Artificial Intelligence, volume 34, pages 8732-8740.

Victor Sanh, Lysandre Debut, Julien Chaumond, and Thomas Wolf. 2019. Distilbert, a distilled version of bert: smaller, faster, cheaper and lighter. arXiv preprint arXiv:1910.01108.

Zhiqing Sun, Hongkun Yu, Xiaodan Song, Renjie Liu, Yiming Yang, and Denny Zhou. 2020. Mobilebert: a compact task-agnostic bert for resource-limited devices. arXiv preprint arXiv:2004.02984.

Nathaniel Weir, Adam Poliak, and Benjamin Van Durme. 2020. Probing neural language models for human tacit assumptions. CogSci.

Jieyu Zhao, Tianlu Wang, Mark Yatskar, Ryan Cotterell, Vicente Ordonez, and Kai-Wei Chang. 2019. Gender bias in contextualized word embeddings. arXiv preprint arXiv:1904.03310.

Jieyu Zhao, Tianlu Wang, Mark Yatskar, Vicente Ordonez, and Kai-Wei Chang. 2018. Gender bias in coreference resolution: Evaluation and debiasing methods. In Proceedings of the 2018 Conference of the North American Chapter of the Association for Computational Linguistics: Human Language Technologies, Volume 2 (Short Papers), pages 15-20, New Orleans, Louisiana. Association for Computational Linguistics.

\section{A Appendices}

\section{A.1 Fine-tuning Experiment}

\section{A.2 Crowdsourcing Instructions}
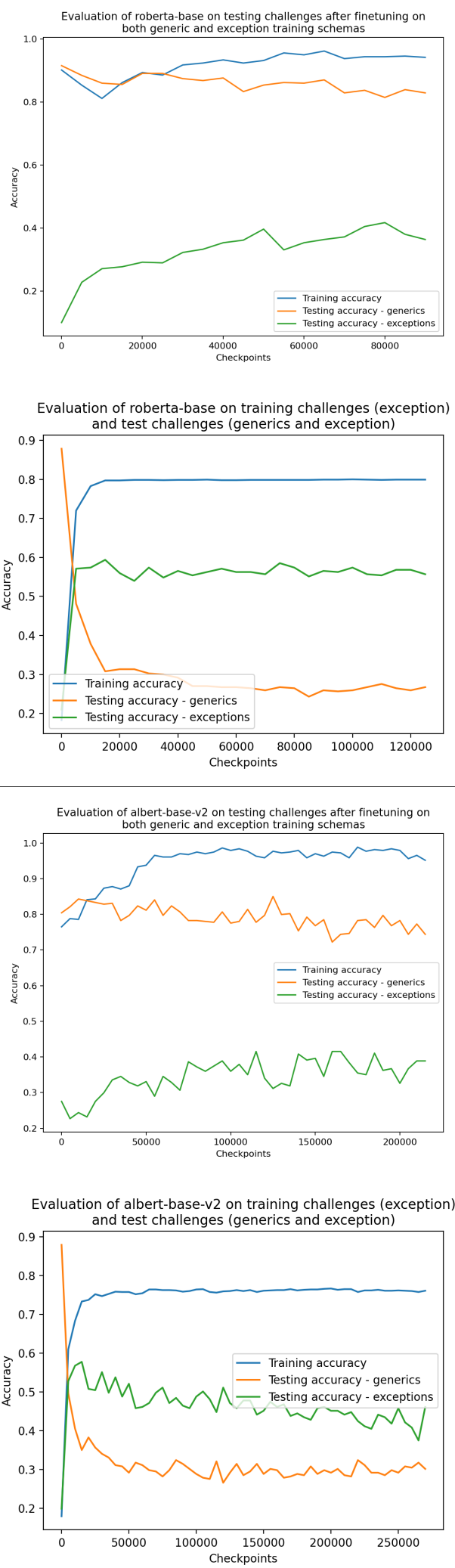

Figure 2: Fine-tuned ALBERT BASE $_{\text {and RoBERTa }}$ BASE show the same negative relationship between generic and exception performances. Fine-tuned RoBERTaBASE shows a higher increase in performance on unseen exceptions in comparison to BERT $\mathrm{BASE}_{\mathrm{BA}}$. 


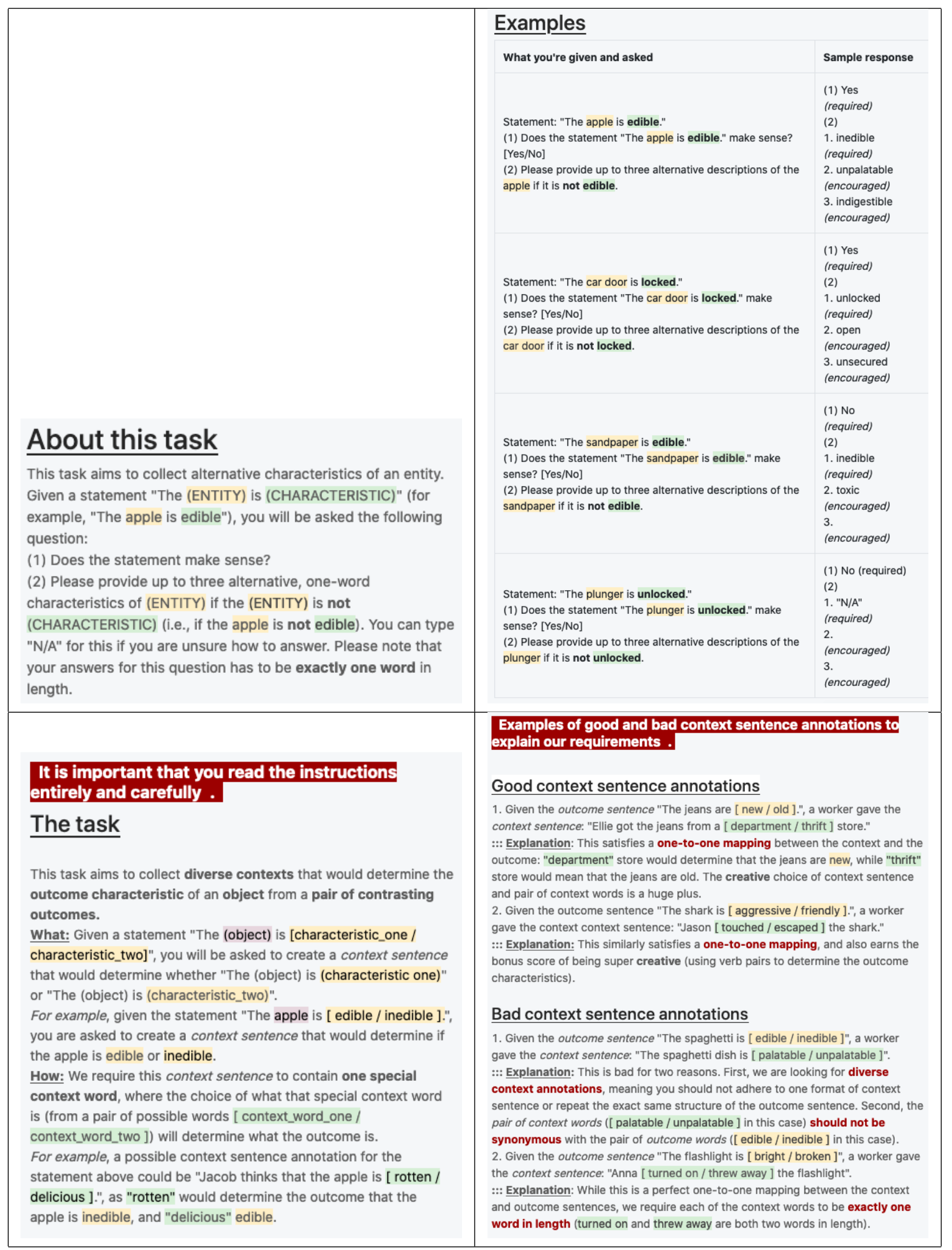

Figure 3: Top Left: Exception Association Collection - Instructions. Top Right: Exception Association Collection - Examples. Bottom Left: Premise Collection - Instructions. Bottom Right: Premise Collection - Examples. 


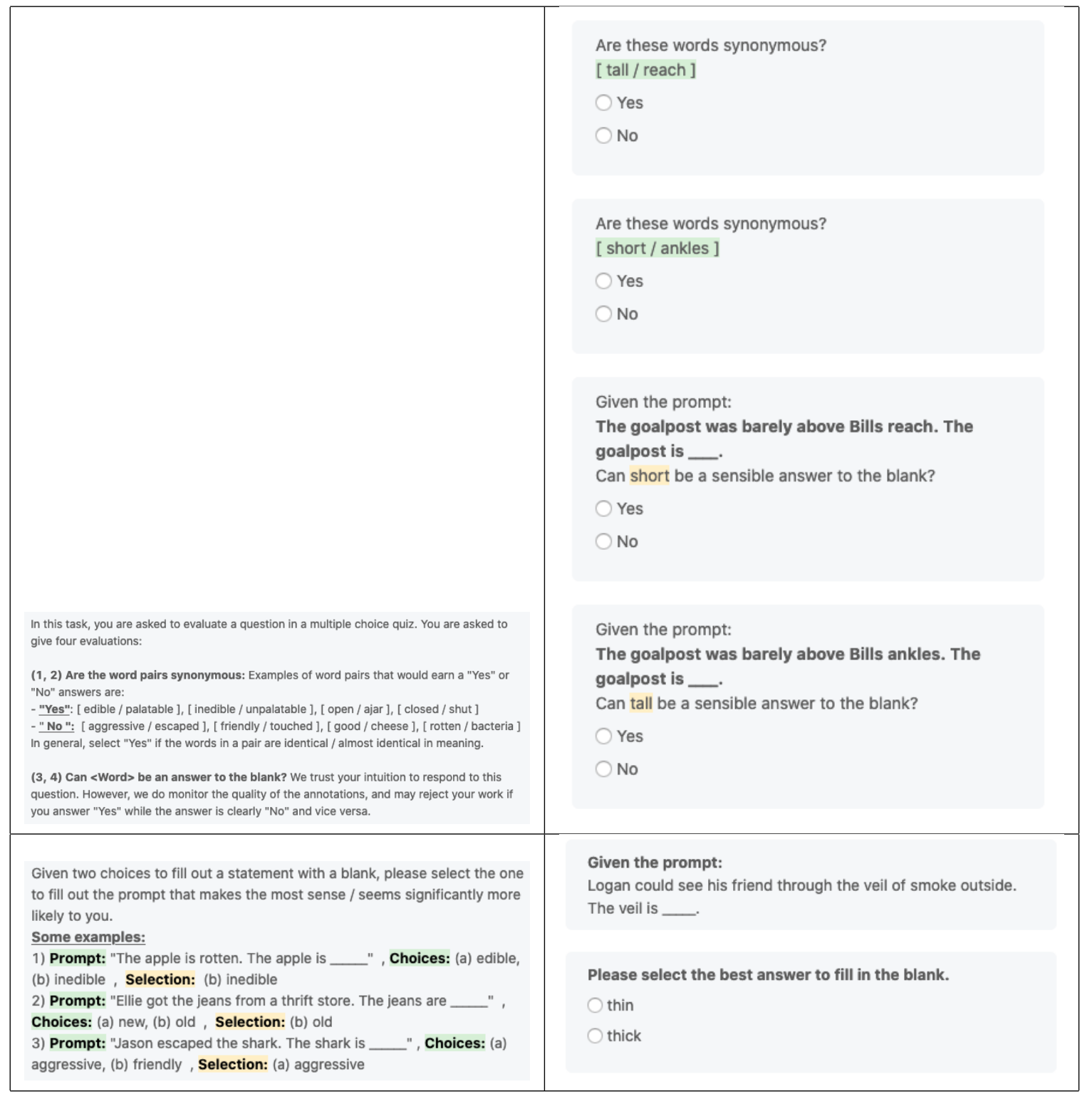

Figure 4: Top Left: Challenge Set Validation - Instructions. Top Right: Challenge Set Validation - Task Sample. Bottom Left: Human Performance Collection - Instruction and Examples. Bottom Right: Human Performance Collection - Task Sample. 